\title{
Patient Allergic to Contrast Media
}

National Cancer Institute

\section{Source}

National Cancer Institute. Patient Allergic to Contrast Media. NCI Thesaurus. Code C69197.

Patient identified to be at risk for an allergic reaction to contrast media. 